\title{
Synchronization of a class of uncertain chaotic systems
}

\author{
Yaopeng Zhou \\ Department of computer \& Science, Wuxi professional college of science and technology, \\ Wuxi,214028,PR China. \\ email: askazyp@vip.qq.com
}

Keywords: chaotic synchronization; Lyapunov-Krasovskii function; time-delay; feedback control.

\begin{abstract}
Synchronization of a class of time-delay chaotic systems with unknown delay time in this paper. The knowledge of the delay time is unknown. Firstly, based on Lyapunov-Krasovskii function, we show that the response chaotic system can synchronize the driven system. The proposed method is tested and verified by a numerical example.
\end{abstract}

\section{Introduction}

Time-delay systems are widely used in engineering, biology, economy, and other fields. Recently, the synchronization of time-delay systems has been received much attention. A lot of methods and techniques have been proposed for such systems [1-4].

The chaos in time-delay systems was firstly found by Mackey and Glass, and then there exist a lot of researchers in this topic [5-9].Unfortunately, the time-delay are assumed to be a known constant time-delay. However, it is impossible to known or measure the time delay exactly in modern society. In engineering practice, the time delay is alway unknow and time variant. In this paper, based on Lyapunov stability theory and LMI, we discuss the problem of slave systems with adaptation to delay parameter for time-delay master systems, where the time-delay constant is unknown exactly. The advantage of this paper is that the exact value of time-delay is unknown.

The rest of the paper is organized as follows. In section 2, a time-delay chaotic system is presented, and a slave system is also designed. Main results are given in section 3 . In section 4 , an example of chaotic system is illustrated to show the effectiveness of the proposed method, followed by some conclusion remarks in section 5 .

\section{Problem formulation}

Consider a chaotic continuous system with time-delay described by:

$$
\dot{x}(t)=A x(t)+B x(t-\tau)+f_{1}(x(t), t)+f_{2}(x(t-\tau), t)
$$

where $x(t) \in R^{n}$ is a n-dimensional state vector of the system, $A, B \in R^{n \times n}$ are constant matrices, $f_{1}, f_{2}: R^{n} \times R^{n} \times R^{+} \rightarrow R^{n}$ are continuous nonlinear functions, are the parameter perturbations, $\tau>0$ is a unknown constant number delay.

In this note, it is assumed that:

(A1)The state vector of the chaotic systems are bounded, $|x(t)| \leq M,|\hat{x}(t)| \leq M$

(A2)There are real numbers $\beta_{i}>0$ such that $\left\|f_{i}(x)-f_{i}(x)\right\| \leq \beta_{i}\|x-y\|$ for any $x, y \in R$

The slave system based on the chaotic system with time-delay (1) is described by

$$
\dot{\hat{x}}(t)=A \hat{x}(t)+B \hat{x}(t-\hat{\tau})+f_{1}(\hat{x}(t), t)+f_{2}(\hat{x}(t-\hat{\tau}), t)+K(x(t)-\hat{x}(t))
$$

where $K \in R^{n \times n}$ is the coupling matrix to be designed to achieve synchronization,

Then, from (1) and (2), the following error system equation is obtained:

$$
\begin{aligned}
& \dot{e}(t)=A e(t)+B e(t-\tau)-B e(t-\hat{\tau})+f_{1}(x(t), t)-f_{1}(\hat{x}(t), t)+f_{2}(x(t-\tau), t)-f_{2}(\hat{x}(t-\hat{\tau}), t)-K e(t) \\
& =A e(t)+B e(t-\tau)+B \int_{-\hat{\tau}}^{-\tau} \dot{\hat{x}}(t+\theta) d \theta+f_{1}(x(t), t)-f_{1}(\hat{x}(t), t)+f_{2}(x(t-\tau), t)-f_{2}(\hat{x}(t-\hat{\tau}), t)-K e(t)
\end{aligned}
$$


$=A e(t)+B e(t-\tau)+B \int_{-\hat{\tau}}^{-\tau} \dot{\hat{x}}(t+\theta) d \theta+f_{1}(x(t), t)-f_{1}(\hat{x}(t), t)+f_{2}(x(t-\tau), t)-f_{2}(\hat{x}(t-\hat{\tau}), t)-$

$\operatorname{Ke}(t)$

Synchronization requires that $\lim _{t \rightarrow \infty}\|x(t)-\hat{x}(t)\|=\lim _{t \rightarrow \infty}\|e(t)\|=0$

\section{Main result}

According to (A3), we can obtain the following inequalities:

$$
\begin{aligned}
& \| f_{1}\left(x(t)-f_{1}(\hat{x}(t))\|=\| f_{1}(e(t)+\hat{x}(t))-f_{1}(\hat{x}(t))\left\|\leq \beta_{0}\right\|(e(t) \|\right. \\
& \| f_{2}\left(x(t-\tau)-f_{2}(\hat{x}(t-\tau))\|=\| f_{2}(e(t-\tau)+\hat{x}(t-\tau))-\right. \\
& f_{2}(\hat{x}(t-\tau))\left\|\leq \beta_{1}\right\| e(t-\tau) \| \\
& \left\|f_{2}(\hat{x}(t-\tau))-f_{2}(\hat{x}(t-\hat{\tau}))\right\| \leq \beta_{1}\|\hat{x}(t-\tau)-\hat{x}(t-\hat{\tau})\|
\end{aligned}
$$

Now the following theorem gives a criterion for the synchronization of the two chaotic systems with time-delay (1) and (2)

Theorem: If there exist the following linear matrix inequalities

$$
A+B B^{T}+\beta_{0} I-K I+I \leq 0
$$

And the updating laws of the estimated parameters in the form of

$$
\begin{aligned}
& \left.\dot{\hat{\tau}}(t)=-\frac{1}{\sigma} M\|B\|+\beta_{1}\right) \alpha(t) \\
& \alpha(t)=\|\dot{\hat{x}}(t)\|
\end{aligned}
$$

where $\sigma$ is a constant to be chosen such that $\hat{\tau}(t) \geq \tau, \forall t \geq 0$.

Then the globally stability at the origin of the error delayed dynamical system implies that the two chaotic systems with time-delay (1) and (2) are globally asymptotically synchronized.

Proof: Choose the Lkf function

$$
V(t)=V_{1}(t)+V_{2}(t)
$$

where $V_{1}(t)=\frac{1}{2} e^{T}(t) e(t)+2 M\left(\|B\|+\beta_{1}\right) \int_{-\hat{\tau}}^{-\tau} d \theta \int_{\theta}^{0} \alpha(t+s) d s+\sigma(\hat{\tau}-\tau)^{2}$

$$
V_{2}(t)=\int_{-\tau}^{0} e^{T}(t+\theta)(I) e(t+\theta) d \theta
$$

The time derivative of $V_{1}, V_{2}$ along the trajectory of equation(3) are $\dot{V}_{1}=e^{T}(t)(A) e(t)+e^{T}(t)(B) e(t-\tau)+$ $e^{T}(t) B \int_{-\hat{\tau}}^{-\tau} \dot{\hat{x}}(t+\theta) d \theta+e^{T}(t) \beta_{0} I e(t)+\left|e^{T}(t) \beta_{1} I e(t-\tau)\right|+\left|e^{T}(t)\right| \beta_{1}\left|\int_{-\hat{\tau}}^{-\tau} \dot{\hat{x}}(t+\theta) d \theta\right|-e^{T}(t) \operatorname{Ke}(t)$

$2 M\left(\|B\|+\beta_{1}\right) \int_{-\hat{\tau}}^{-\tau}(\alpha(t)-\alpha(t+\theta)) d \theta+2 M\left(\|B\|+\beta_{1}\right) \cdot \dot{\hat{\tau}} \int_{-\hat{\tau}}^{0} \alpha(t+s) d s+2 \sigma(\hat{\tau}-\tau) \dot{\hat{\tau}}$

$\left.\leq e^{T}(t)\left(A+\beta_{0} I-K\right) e(t)+e^{T}(t) B B^{T} e(t)+e^{T}(t-\tau)(I) e(t-\tau)+\left|e^{T}(t)\right| \mid\|B\|+\beta_{1}\right)$.

$\int_{-\hat{\tau}}^{\tau} \mid \dot{\hat{x}}(t+\theta) d \theta+2 M\left(\|B\|+\beta_{1}\right) \cdot \alpha(t)(\hat{\tau}-\tau)-2 M\left(\|B\|+\beta_{1}\right) \int_{-\hat{\tau}}^{\tau} \alpha(t+\theta) d \theta+2 \sigma(\hat{\tau}-\tau) \dot{\hat{\tau}}$

Substituting (6) (7), into (10), one can obtain that

$\dot{V}_{1}(t) \leq e^{T}(t)\left(A+\beta_{0} I-K+B B^{T}\right) e(t)+e^{T}(t-\tau)(I) e(t-\tau)$

also we can obtain that

$\dot{V}_{2}=e^{T}(t)(I) e(t)-e^{T}(t-\tau)(I) e(t-\tau)$

Substituting (9) into (10) gives that

$\dot{V}_{1}+\dot{V}_{2} \leq e^{T}(t)\left(A+B B^{T}+\beta_{0} I-K+I\right) e(t)$ 
As we know that $A+B B^{T}+\beta_{0} I-K+I$ is negative, it is easy for us to obtain that two chaotic systems with time-delay (1) and (2) are globally asymptotically synchronized.

\section{Illustrative example}

To demonstrate the effectiveness of chaos synchronization criterion proposed herein, a chaotic system is considered:

$\zeta \frac{d x}{d t}=-(A) x(t)+\frac{G}{1+\mu}\left(x(t-\tau)+U_{B}\right)\left[1+\mu \cos \left(\pi\left(x(t-\tau)+U_{0}+U_{M}\right)\right]\right.$

where $x(t)$ is a state vector of the chaotic system, $G \geq 0$ is a feedback gain constant, $h \geq 0$ is a unknown constant number delay. $\mu \geq 0, \varsigma \geq 0, U_{B}, U_{0}, U_{M}$ is the constants of the chaotic systems. where $A=1, \mu=1$

$U_{0}=2.0, U_{B}=1.0, U_{M}=-2.5, G=2.0, \varsigma=0.1, \tau=0.01$.

According to the theorem, we can obtain $K=5$, then the errors between two chaotic systems as shown in Fig.1.

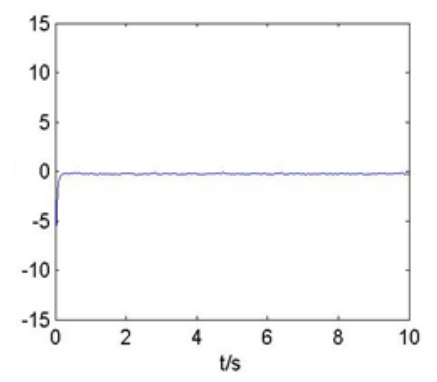

Fig1.The diagram present the error $e(t)$ between the two chaotic systems.

\section{Conclusion}

In this paper, we investigated the time-delay master-slave chaos synchronization of two coupled delayed systems using the unidirectional linear error feedback scheme. Compared with the existing literatures, the delay time of the master system is no required to be known, and this method can be extended to the systems comtained time variant delay.

\section{References}

[1] Lin J S, Liao T L, Yan J J, Yan H T. Synchronization of unidirectional coupled chaotic systems with unknown channel time-delay: Adaptive robust observer-based approach [J]. Chaos, Solitons \& Fractals 2005; 26(3):971-978.

[2] Tong H K. Eun T J. Hong B P. Robust control for parameter uncertain delay systems in state and control input [J].Automctic, 1996, 32(9):1337-1339.

[3] Young S M, Park P G, Wook H K. Robust stabilization of uncertain input-delayed systems using reduction method[J].Automatic,2001,(2):307-312.

[4] Yue D. Robust stabilization of uncertain systems with unknown input delay [J].Automatic, 2004, 40(2):331-336.

[5] Yue D, Han Q L. Delayed feedback control of uncertain systems with time-varying input delay [J].Automatic, 2005, 41(2):233-240.

[6] Jiang X F, Xu W L, Han Q L. Observer-based fuzzy control design with adaption to delay parameter for time-delay systems[J].Fuzzy sets and systems,2005,152(3):637-649.

[7] Ott E, Grebogi C, Yorke J A. Controlling chaos [J]. Phys Rev Lett, 1990, 64: 1196-1199.

[8] Dressler U, Nitsche G. Controlling chaos using time delay coordinates [J]. Phys Rev Lett ,1992,68:1-4

[9] Tsai H H, Fuh C C, Chang C N. A robust controller for chaotic systems under external excitation [J]. Chaos, Solitons \& Fractals, 2002, 14: 627-632. 